\title{
Increasing luminous efficiency of led by joint dimming mode with adjustable peak current
}

\author{
Yongtao.Li, Jierong.Zhao, Youran.Li, Yuncui.Zhang, Nianyu.Zou and Jinpeng.Wang ${ }^{\text {a }}$ \\ Research Institute of Photonics, Dalian Polytechnic University, 116034 Dalian,China
}

\begin{abstract}
Luminous efficiency and color temperature of LED chip are affected by current and temperature. PWM dimming mode only works at two kinds of current levels-rated current and zero current. It has smaller effect on color temperature compared with linear dimming mode, but it will reduce the luminous efficiency of lamps. Linear dimming mode has higher luminous efficiency by comparison. The two dimming modes above cannot keep color temperature stable and Luminous efficiency high. Therefore, the paper designed a joint dimming mode by integrating advantages of the two modes. The dimming method not only adjusts peak current, but also changes the duty cycle of dimming signals. The injected average current is kept stable by reducing peak current and increasing the duty cycle. The decreased luminous efficiency by the raise of temperature was made up for. Then according to the mathematical model of total flux and current, the author calculates the mathematical mode of flux affected by temperature. It proves the fact that adjusting peak current slightly can compensates for the reduced luminous efficiency affected by temperature through theoretical calculation. In addition, the final peak current after calculation can contribute to the adjustment parameter of joint dimming modes.
\end{abstract}

\section{Introduction}

The technology of semiconductor develops constantly, and significant changes have taken place in illuminating lamps [1]. LED luminaire products are becoming more and more popular. As people have a higher demanding of illuminating environment, dimming technology emerges so as to provide a proper environment for people [2].Such as J. J. Zhang analysed radiant efficiency and thermal analysis of high-power LEDs at linear and PWM dimming modes and got that linear dimming mode's radiant efficiency is higher than PWM dimming mode when input power is same [3], X. S. Fu designed a novel isolating PWM dimming LED driver and the result showed that power exceed 0.9 , the efficiency exceed $85 \%$, and the dimming range of $2 \% \sim 100 \%[4]$. And X. B. Jiang designed the device of analog dimming mode on ZigBee technology, it realized the cooperation of the ZigBee internet gateway, monitoring and controlling remotely[5]. X. H. Qu proposed a led current sharing circuit with high luminous efficacy and individual dimming; it could adjust independently and improve the luminous efficiency [6]. Combining Standard for lighting Design of Urban Road and taking account of lighting comfort, it adjusts lighting parameters by changing output current, output power and duty cycle[7-9]. Theories of the technology include TRIAC dimming mode, partition dimming mode, linear dimming mode and PMW dimming mode [10-12]. However, there are some problems in the practical application of the above theories [13]. For example, there is the problem about that the output of linear dimming is not very dexterous, and the dimming-dynamic range is narrow [14-17].Traditional

\footnotetext{
a Corresponding author: wangjp@dlpu.edu.cn
}

PMW dimming can caused electromagnetic interference frequency, and produced color cast as the temperature rises[18].

The paper combined the small effect PMW dimming mode had on color temperature with the advantage of high luminous efficiency of analog dimming, proposing a joint dimming mode based on these two dimming modes. The new mode not only kept color temperature of the lamps stable, but also made luminous efficiency remain at a high level. By looking up luminous flux and changing trend of current of a lamp in OSRAM Company, the author established a mathematical model related to them, and gave the compensation current through calculating. So it provided reference for adjusting current.

\section{The traditional dimming mode}

The TRIAC dimming mode achieved the function of dimming through silicon controlled rectifier dimmer working with the drive circuit. The dimming mode was commonly anywhere. It mainly was used on thermal radiation source, such as the incandescent lamp. It would redesign the driver circuit if the dimming mode was used on LED. There are some problems that LED lamp flickered when it was dimming, the illuminance was not balance, and it would produce the audio-frequency noise.

Analog dimming technology is called linear dimming; it changes the injected forward current by adjusting the parameters of potentiometer in peripheral circuit. Then the lamp's illuminance level is adjusted. The dimming mode had the following advantages, such as simple peripheral circuit, controllable, high luminous efficiency 
and it could not introduce the electromagnetic interference in operation. But the color temperature drift was obvious because the junction temperature was raised when it adjusted the current in the wide range.

Partition dimming mode was using switch frequency in a certain time to adjust the illuminance of lamp. For example, a panel switch can be changed three sections. The first time of pressing down was full bright; the second was the percent of seventy-five of full light; the third was the percent of fifty of full light; the forth was the percent of twenty-five of full light. The dimming mode did not need the extra wiring, but it could not adjust illuminance continuously.

PWM is abbreviation of the Pulse Width Modulation.it kept the rated current in steady-state, changing the average current by adjusting the duty cycle in dimming operation. So it achieves the objective of dimming. The dimming mode is working in two types of current, such as the rate current and the zero current. So the color temperature drift is stable compared with linear dimming mode. Generally speaking, it used the physiological characteristics of the human eyes needing a short adjustment time to make them not detect to the light constantly blinking. And the frequency of dimming signal in operating is set to $200 \mathrm{HZ} \sim 20 \mathrm{KHZ}$. But it causes the ceramic capacitor to make noise in the circuit if the dimming signal is working in the high frequency. At the same time, the dimming circuit introduced the additional PWM signal generating module leading to be more complex. It was very bad that the luminous efficiency reduced compared with linear dimming mode.

Designing a new dimming mode is impending. The functions are shown following: high luminous efficiency, stable color temperature and dimming range from $0 \sim$ $100 \%$. So the joint dimming mode was designed for realizing the functions above.

\section{The design of joint dimming mode}

The signal generating circuit's core chip is the PT4115. The chip integrated the EMI filtering function to ensure that the circuit is not affected by jamming of harmonic in the grid. And the chip's input voltage range from $8 \mathrm{~V}$ to 30V.It integrated the DC/DC-BUCK highly to keep the output current constant. The circuit is integrated with the LED display module. It displayed the value of the duty cycle facilitating adjusting the effective-average current. The pins of chip packaging are shown in Figure 1.

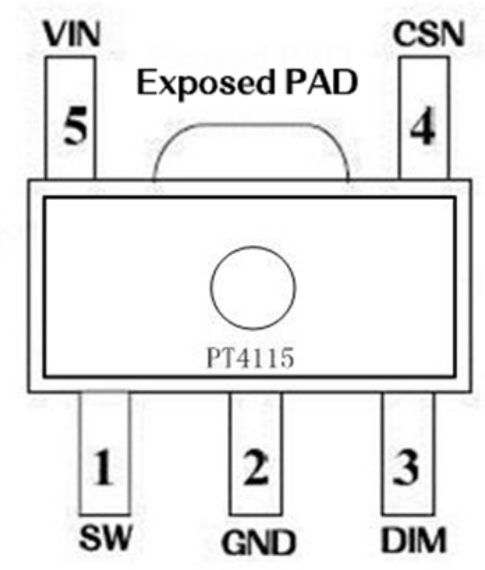

Figure 1.The package pin map of PT4115.

The function of all pins is as follows: 1.SW: Switch Output, SW is the drain of the internal N-CH MOSFET switch; 2.GND: Signal and power ground, connect directly to ground plane; 3.DIM: Logic level dimming input. Drive DIM low to turn off the current regulator. Drive DIM high to enable the current regulator; 4.CSN: Current sense input; 5.VIN: Input Supply Pin. Must be locally by passed;

The PT4115 is the core chip of the dimming circuit as same as the signal generating circuit. And the PWM dimming circuit is a constant-current driver. The PWM signal is loaded into the DIM pin of dimming module in operation, as shown in Figure 2 (a). It will turn off LED current, when the PIN voltage is less than $0.3 \mathrm{~V}$, and it will open the LED current, if the PIN voltage is more than $2.5 \mathrm{~V}$.

The joint dimming mode combined the advantages of the analog dimming and PWM dimming modes. It kept not only color temperature stable but also luminous efficiency remaining in a high level. The joint dimming mode increased luminous efficiency by reducing the peak current. The potentiometer was connected between the DIM and GND pins to adjust the peak current, as shown in Figure 2 (a). The dimming module was adjusted average current by changing duty cycle of the dimming signal, when it kept the potentiometer invariable.

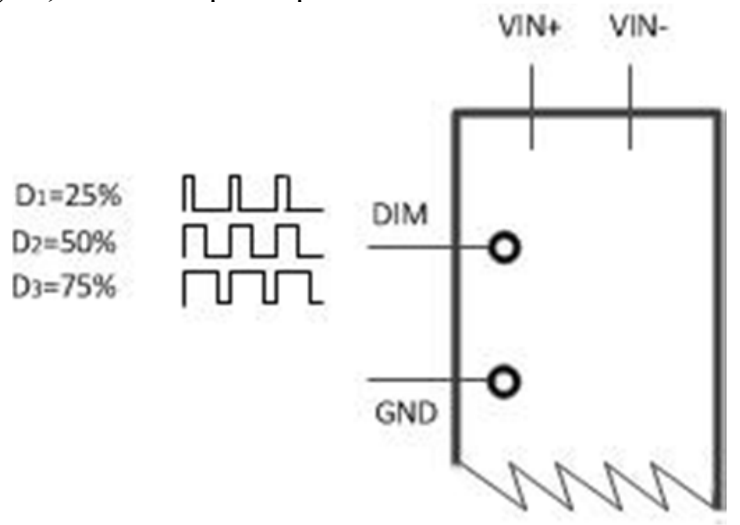

(a) PWM dimming signal access local. 


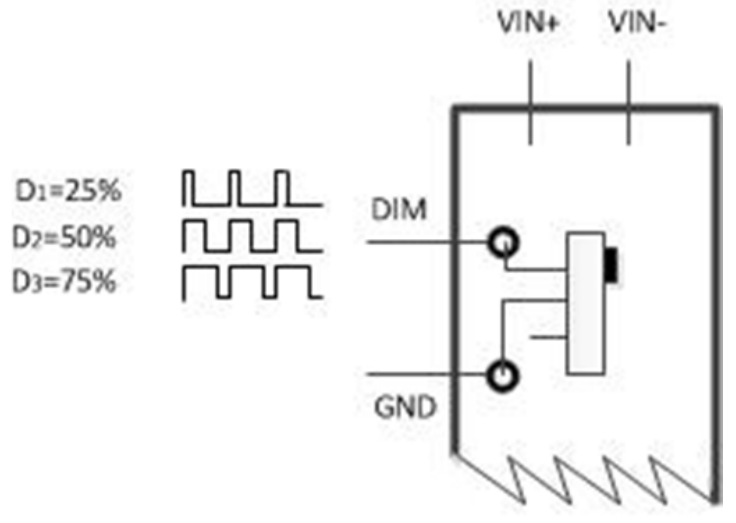

(b) Joint dimming mode's pin access local.

Figure 2.The local pin map of two dimming modes.

Generally speaking, a dimming mode only adjusted one parameter. But the joint dimming mode could adjust the duty cycle and peak current independently by improving the original PWM dimming circuit. So it kept the average input power constantly. The relation between duty cycle and peak current is expressed 1 .

$$
I_{\text {avg }}=I_{\text {peak }} * D
$$

$I_{\text {avg }}$ is the average-output current, $I_{\text {peak }}$ is the peak current , $D$ is the duty cycle of PWM signal. It could be known that there was the inverse relation between peak current and duty cycle when the average current was constant. The duty cycle would decrease when peak current increased and the peak current would decrease when the duty cycle increased.

\section{Establishing Mathematical Model}

The luminous efficiency of white LED would reduce when the current increased. Because, all parameters will be affected by the rose temperature, such as electron mobility reducing, the radiative recombination rate of electrons and holes in the well trap reducing. The luminous efficiency would be affected by the factors. But the effect of temperature on luminous efficiency was reversible. It says that the luminous efficiency has a tendency to recover, when the temperature decreases. The main factors of influencing luminous efficiency are current and temperature, so the paper just considers the effects of current and temperature. It got the relation between luminous efficiency and current by analysing the relationship between flux and current. Then it compensated the luminous efficiency to make it remain at a high level by the joint dimming mode.

It got the trendy of flux and forward current by analysing a lamp of the OSRAM, as shown in Figure 3(a). According to information, the relation between the flux and current was linear without other influencing factors. But the effect of temperature increased gradually with the increasing current. It led to reducing the growth rate of flux, decreasing the luminous efficiency. The front of curve was linear with the increased current. But the slope would reduce with the increased current when the current increased to a certain value. Therefore the relation for actual measured data between flux and current was consistent with the trend of theoretical analysis. The choice of lamp was random, so the analysis about the luminaire was universal. It can reflect the characteristics of the most LEDs.

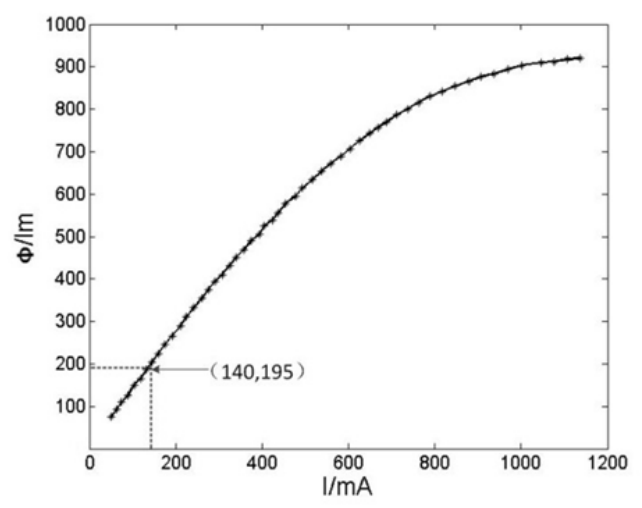

(a) The relation between and current.

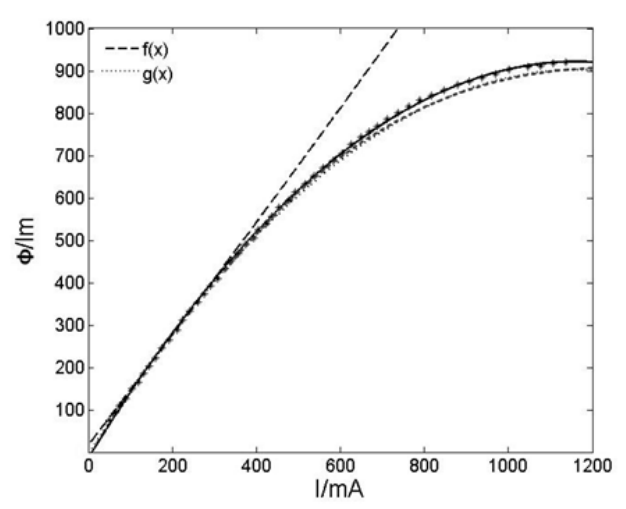

(b) Mathematical model of piecewise function.

Figure 3.The local pin map of two dimming modes.

It could be seen an obvious Cut-off point between the line and curve. The coordinates of the Cut-off was $(140,195)$. So we could build a mathematical model of piecewise function, as shown in Figure 3 (b). It got a linear relationship by fitting the sampling points at the front of Curve, the relation was expressed 2. The relation between flux and current was nonlinear when the current was greater than the cut-off value. It got a nonlinear relation by fitting the sampling points, the relationship was expressed 3.

$$
\begin{gathered}
f(x)=\mathrm{b}_{1} * x+\mathrm{c}_{1} \\
g(x)=\mathrm{a}_{2} * x^{2}+\mathrm{b}_{2} * x+\mathrm{c}_{2}
\end{gathered}
$$

The expression of piecewise function was shown 4 .

$$
T(x)=\left\{\begin{array}{cr}
b_{1} * x+c_{1} & , x<140 \\
a_{2} * x^{2}+b_{2} * x+c_{2}, & x \geq 140
\end{array}\right.
$$

The reasons for reducing luminous efficiency were caused by temperature. The relation between flux and temperature was got by adding equation 1, 2 together. It was expressed 5 .

$$
h(x)=\mathrm{a}_{3} * x^{2}+\mathrm{b}_{3} * x+\mathrm{c}_{3}
$$


The parameters of fitting equation were calculated by the sampling points. Taking the sampling points' coordinated into the mathematical model, it got the best degree of fitting as original data. So it could calculate the parameters. They were shown in table 1 .

Table 1. the parameters of fitting equation.

\begin{tabular}{|c|c|c|c|}
\hline $\begin{array}{r}\text { equation } \\
\text { s subscripts }\end{array}$ & $\mathrm{a}$ & $\mathrm{b}$ & $\mathrm{c}$ \\
\hline 1 & $/$ & 1.34 & 8.97 \\
\hline 2 & $-0.66 \times 10^{-3}$ & 1.55 & -5.41 \\
\hline 3 & $0.66 \times 10^{-3}$ & -0.21 & 14.38 \\
\hline
\end{tabular}

It got the relationship between flux and current by analyzing the mathematical model above. And it got the relation between flux and temperature too. The compensation current of was calculated. It kept the range of flux same for increasing the luminous efficiency by reducing the peak current, as expressed 6 .

$$
\triangle f(x)=\triangle h(x)
$$

Assuming the range of flux was $(652,689)$, it could get the $(537,583)$ of $g(x)$ and the $(479,505)$ of $f(x)$. It knew that the results of calculation were consistent with theoretical data. It kept luminous efficiency remaining in a high level by reducing the peak current.

According to the calculated range of current, luminous efficiency could be increased by joint dimming mode. It calculated the value of duty cycle by equation 1 , so as to increase the luminous efficiency.

\section{Conclusions}

The author focused on the relationship between luminous effect and current, and the relationship between luminous efficiency and temperature. A method of increasing luminous efficiency through reducing peak current was proposed. It was based on traditional dimming mode, such as linear dimming mode and PWM dimming mode. And a joint dimming mode was proposed by combining high luminous efficiency of analog dimming with the advantage of stable color temperature PWM dimming possesses. The new dimming mode could change the duty cycle of dimming circuit, and adjust peak current of circuit to achieve the function of adjusting two parameters-duty cycle and current independently. Then it established a related piecewise function by the relation between flux and current. The piecewise function was made up of the part of linear relation and the part of curve relation. There was an obvious Cut-off point. Then it got the mathematical model about luminous efficiency and temperature by calculating. The required current was calculated, and it was the reference of the joint dimming mode.

\section{Acknowledgements}

This research was supported by Project of the National Natural Science Foundation of China (61402069), 2012 key supported project for undergraduate education by Education Dept. of Liaoning province( [2012]108), 2016 Project of the Natural Science Foundation of Liaoning province(2016020027), 2012 Education reform project for undergraduate by Education Dept. of Liaoning province ( [2012]130), 2014 Dalian municipal science and technology plan project (2014A11GX050), 2014 Dalian municipal science and technology plan project(2014A11GX052).

\section{References}

1. H.M. Chen, Semiconductor.Technol.35,101(2010)

2. Z. L. Liu, Z. N. Guo, Z. W. Hu, J. HuaQiao Uni, 34, 14(2013)

3. J. J. Zhang, T. Zhang, Q. Zheng, J .OELE - Laser. 24,50(2013)

4. X. S. Fu, T. Wang, M. Q. Wang TRA. CHI .ELETECHN.SOC,30,75(2013)

5. X. B. Jiang, K. Han, H. P. Chen, ZM.GONGCHEN $\mathrm{G} \mathrm{XB,} \mathrm{21,} 4$ (2010.)

6. X. H. Qu, Y. P. Tang, S. C. WONG, Proc. CSEE, 34, 1(2014)

7. R. Feng, C. Z. J. Ge, L. Zhu, JX S, 29,424(2011)

8. P. Qian, S. Zheng, H. Wang, M. Chen, POW. Electron. 44, 3,( 2010)

9. J. Wang, X. Huang, L. Liu, CHI J LUMINE, 29, 358(2008)

10. J. M. Alonso, D. G. Vaquero, IEEE. Trans Indu. Elec, 59,1689(2012)

11. C. D. Bi, F. H. Zhang, B. A. Qi, J SHANXI. UNI \& TEC 29,56(2011)

12. P. C. Song, S. S. Wen, W. P. Guan, J Opt. elec. Laser, 26,1895(2015)

13. Z.Vaitonis,A.Stonkus,A.Žukauskas, IET .OPT.ELEC, 6, 52(2012)

14. Y.L. Lin, J. Huang, IND Elec. IEEE, 61,4632(2014)

15. S. K. Jin, J. A. Yoon,Opt.Mat,47, 78(2015)

16. C. J. Tian, X. Y. Zhang, J. Zou, CHI. J LUMIN, 31, 96(2010)

17. C.Terry, EC\&M. Elec. Cons \& Main, 114, 10(2011)

18. Q. B. Feng, H. J. He, D. Han, Opt Laser Technol, 77, 106(2015) 